\title{
INTRALUMINAL GOSSYPIBOMA - A RARE CASE PRESENTATION
}

\author{
Pankaj Kumar Sarkar1, Shibaji Basu², Shibsankar Roychaudhuri ${ }^{3}$
}

${ }_{1}^{1}$ Assistant Professor, Department of General Surgery, Medical College Hospital, Kolkata.

${ }^{2}$ RMO, Department of General Surgery, Medical College Hospital, Kolkata.

${ }^{3}$ Associate Professor, Department of General Surgery, Medical College Hospital, Kolkata.

\section{ABSTRACT}

Gossypiboma, an infrequent but serious surgical complication with medico-legal implication, is a mass lesion due to a retained surgical sponge surrounded by foreign-body reaction. A 70-year-old lady presented with palpable abdominal mass two years after abdominal hysterectomy done through lower midline incision. Retained foreign body was one of the differential diagnosis, though exact diagnosis could not be clinched despite aggressive investigations. Retained foreign body should be in the differential diagnosis of any postoperative patient who presents with pain, infection or palpable mass in which no diagnosis could be reached clinically as well as radiologically.

\section{KEYWORDS}

Previous laparotomy; Retained Foreign Body (RFB); Intraluminal Gossypiboma; Palpable abdominal lump; CECT abdomen (Contrast Enhanced Computerised Tomography)

HOW TO CITE THIS ARTICLE: Sarkar PK, Basu S, Roychaudhuri S. Intraluminal gossypiboma - a rare case presentation. J. Evolution Med. Dent. Sci. 2016;5(87):6523-6525, DOI: 10.14260/Jemds/2016/1475

\section{BACKGROUND}

A surgical sponge is one of the most common type of Retained Foreign Body (RFB). The condition is sometimes called gossypiboma, derived from the Latin gossypium (Cotton) and the Swahili boma (Place of concealment). Two usual responses lead to the detection of a retained sponge. The first type is an exudative inflammatory reaction with the formation of an abscess and usually leads to early detection and surgical removal. The second type is aseptic with a fibrotic reaction to the cotton material and development of a mass.[1]

In the abdomen the sponge can be surrounded by omentum and intestines, which attempt to encapsulate it. The exerted pressure and irritation on the bowel loops can lead to necrosis of the intestinal wall and the sponge erodes partially or entirely into the lumen of the bowel. This process can lead to obstruction or fistula. Patients develop symptoms of abdominal pain, nausea, vomiting, anorexia and weight loss resulting from obstruction or a malabsorption type syndrome caused by the multiple intestinal fistulas or intraluminal bacterial overgrowth.[1,2]

\section{CASE PRESENTATION}

A 70-year-old lady presented with discomfort and a swelling in hypogastric area for the last five months. Apart from that there were no complaints. Bowel and bladder habits were normal. The only positive point in her past history was an abdominal hysterectomy done two years back through lower midline incision and open cholecystectomy thirteen years back through right subcostal incision. Vital signs were normal.

Financial or Other, Competing Interest: None.

Submission 25-09-2016, Peer Review 19-10-2016,

Acceptance 25-10-2016, Published 29-10-2016.

Corresponding Author:

Dr. Pankaj Kumar Sarkar,

Assistant Professor,

\#334/4, Dr. M. N. Saha Road,

Dumdum, Kolkata-700074.

E-mail: pankajkrsarkarl5@gmail.com,

surgeon_pankaj_sarkar75@yahoo.com

DOI: $10.14260 /$ jemds/2016/1475

\section{(c) $(1) \ominus$}

On abdominal examination, a round mobile intraabdominal mass was palpable in the hypogastrium and left iliac fossa, soft in consistency, but irregular in outline. Digital rectal examination was normal. All routine lab investigations were normal apart from decreased haemoglobin. CECT whole abdomen was not confirmatory. It had three differential diagnoses: chronic intussusception, retained foreign body (? mop) or bezoar. Full length colonoscopy was also normal. Diagnostic laparoscopy before exploratory laparotomy revealed only a thickened and distended part of ileum and caecum; normal looking liver without any ascites or peritoneal swelling.

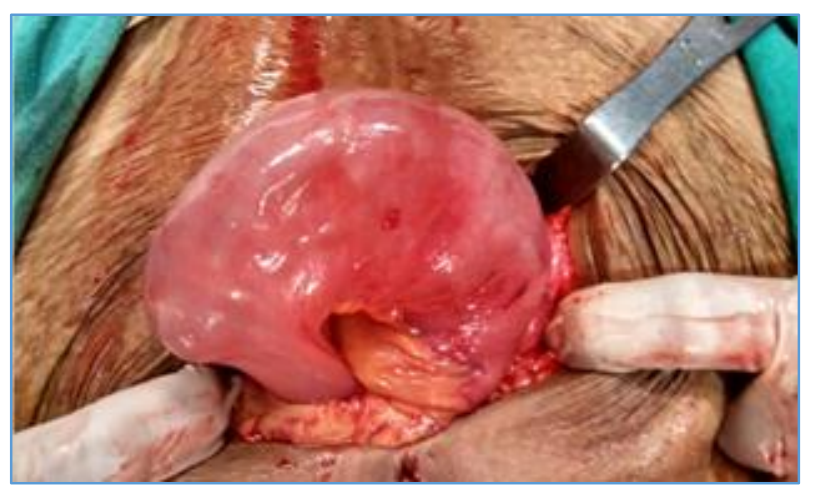

Figure 1. Intraluminal Gossypiboma after Laparotomy

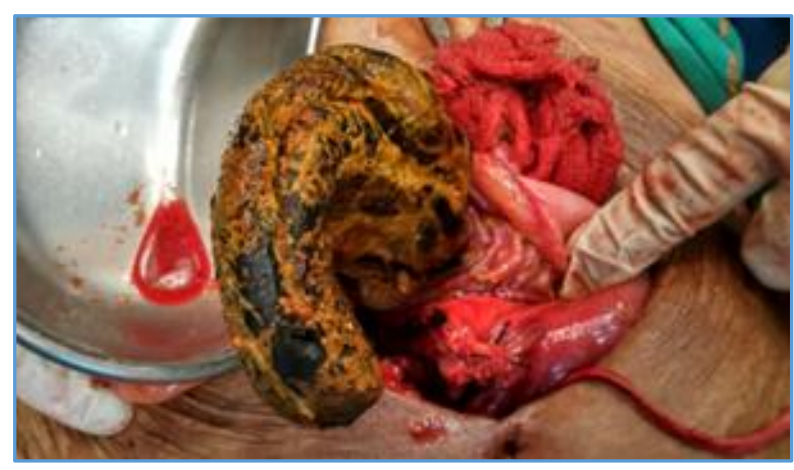

Figure 2. Intraluminal Gossypiboma seen Coming Out After Cutting the Intestine 


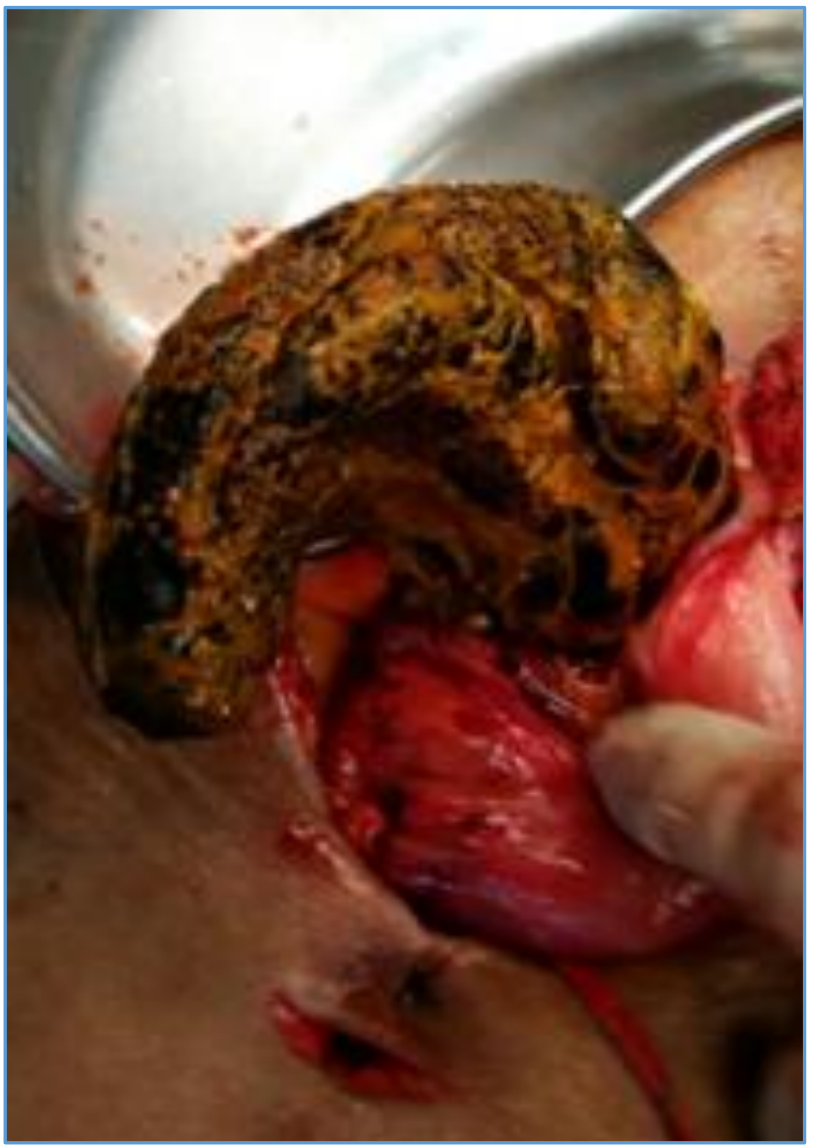

Figure 3. Intraluminal Gossypiboma Coming Out After Cutting Intestine

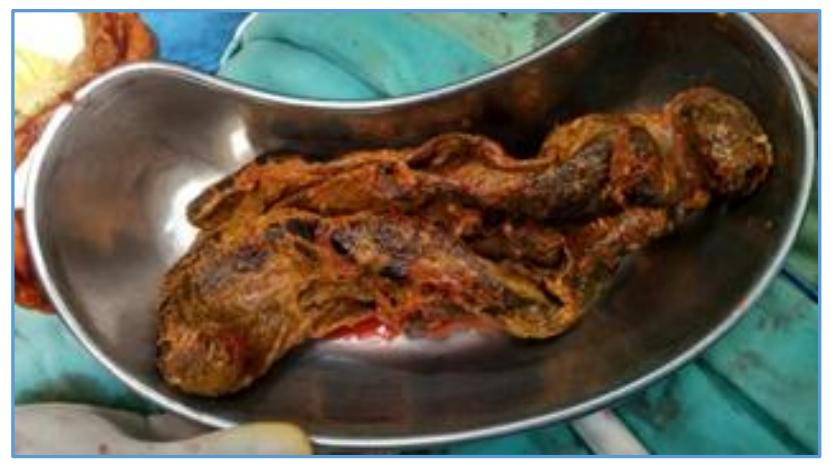

Figure 4. Gossypiboma after Complete extraction in Toto

A limited resection of the involved segments were done and ileoascending anastomoses performed. Exploration of the bowel specimen revealed an intact mop with tail. Postoperative course was uneventful.

\section{DISCUSSION}

The possibility of an RFB should be in the differential diagnosis of any post-operative patient who presents with pain, infection or palpable mass. The first diagnostic modality to rule out an RFB should be a CT scan and often it will be the only test needed. The CT findings of a sponge usually describe a rounded mass with a dense central part and an enhancing wall. Other features of retained sponges or towels include a whorllike appearance with trapped air bubbles and cystic masses with infolded densities. MRI features can be confusing because the radio-opaque marker is not magnetic or paramagnetic, so is not visible.[1]
Clinicians usually think that the diagnosis of an RFB on an intraoperative radiograph is easy and obvious, but often this is not the case. Intraoperative radiographs can be of poor quality, especially in obese patients. Correctly identifying a sponge on a radiograph can be difficult. The surgical markers may become twisted or folded and present an unusual image.[3] For instance, in a report of 13 patients with a retained sponge the radio-opaque marker inside the sponge was seen in only 9 radiographs and even then was not immediately recognised for what it was.[4] Markers have been misinterpreted as calcifications, intestinal contrast material, wires or surgical clips.[1]

The usual treatment of an RFB is removal. Reopening the previous operative site is one possibility, but endoscopic or laparoscopic approaches may be attempted.[5]

One possible complication during surgical removal of RFB is perforation of adherent bowels, which may be missed.

In some instances, the attempt to remove the retained foreign body may cause more harm than the item itself, although in these circumstances the foreign body is usually a needle or small part of a surgical item. In these cases, removal is not recommended. Rarely is this an appropriate course of action for a retained sponge, which should always be removed.[1]

Recently, New England Journal of Medicine published an article about risk factors of RFBs. Of the 8 risk factors, the authors identified (emergency operation, unexpected change in operation, more than one surgical team involved, change in nursing staff during procedure, body mass index (BMI), volume of blood loss, female sex and surgical counts) only 3 were found to be statistically significant by multivariate logistic regression. The 3 significant risk factors were emergency surgery, unplanned change in the operation and BMI. The counting of sponges and instruments was not a significant predictor in the multivariate model. Although, all 3 factors were significant, the 9-fold increase in risk associated with emergency surgery was impressive.

In addition, in $88 \%$ of the cases where there was an RFB and counts were performed, the counts were falsely called correct. The authors recommended "radiographic screening" at the end of high risk cases as a possible adjunct to improve detection of RFB.[6] Surgeons should place radiologically detectable sponges and towels in the surgical site, carefully considering the use of small sponges in large cavities and perform a methodical wound examination each and every time before they begin to close the wound.[1]

New technologies are being developed that will hopefully decrease the incidence of RFB. An electronic article surveillance system has been examined, which uses a tagged surgical sponge that can be identified electronically.[7] Bar codes can be applied to all sponges and with the use of a bar code scanner the sponges can be counted on the back table. The use of radiofrequency identification system holds much hope for application in the area of detection of sponges. ${ }^{[1]}$

\section{CONCLUSION}

RFB should be considered in the differential diagnosis of any postoperative patient who presents with pain, infection or palpable mass. Identifying a sponge on an intra-operative radiograph is difficult. The best diagnostic modality to rule out an RFB should be a CT scan or diagnostic laparoscopy. But in rare case of intraluminal RFB, only exploratory laparotomy 
confirms the diagnosis. One possible complication during surgical removal of RFB is missed perforation of adherent bowels.

\section{REFERENCES}

1. Gibbs VC, Coakley FD, Reines HD. Preventable errors in the operating room: retained foreign bodies after surgery. Curr Probl Surg 2007;44(5):281-337.

2. Cruz RJ, de Figueiredo PLF, Guerra L. Intracolonic obstruction induced by a retained surgical sponge after trauma laparotomy. J Trauma 2003;55(5):989-91.

3. Revesz G, Siddiqi TS, Buchheit WA, et al. Detection of retained surgical sponges. Radiology 1983;149(2):411-3.
4. Kopka L, Fischer U, Gross AJ, et al. CT of retained surgical sponges (textilomas): pitfalls in detection and evaluation. J Comput Assist Tomogr 1996;20(6):919-23.

5. Karahasanoglu T, Unal E, Memisoglu K, et al. Laparoscopic removal of a retained surgical instrument. J Laparoendosc Adv Surg Tech 2004;14(4):241-3.

6. Gawande AA, Studdert DM, Orav EJ, et al. Risk factors for retained instruments and sponges after surgery. $\mathrm{N}$ Engl J Med 2003;348(3):229-35.

7. Fabian CE. Electronic tagging of surgical sponges to prevent their accidental retention. Surgery 2005;137(3): 298-301. 